\title{
Tifosi o hooligan? Miti e stereotipi del tifo inglese
}

\author{
di Gianni Silei
}

Giu 3, 2021 | In evidenza, La storia dello sport $|\underline{0}|$

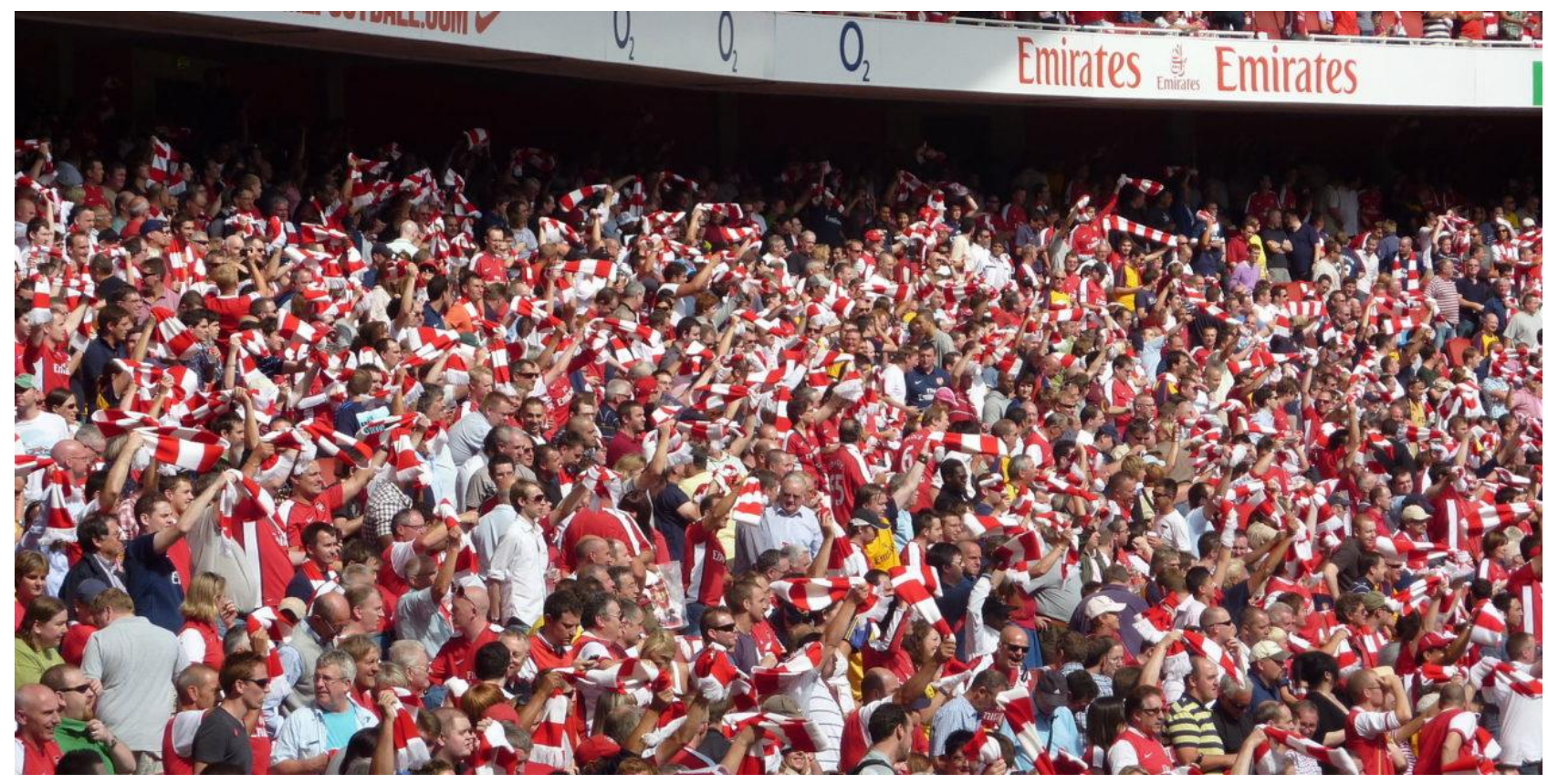

I tifosi della squadra londinese dell'Arsenal.

Foto di wonker - https://www.flickr.com/photos/wonker/3845938367/in/set-72157622113288986/, CC BY 2.0, Link

\section{Abstract}

Malgrado le immagini degli stadi di calcio inglesi mostrino ormai da qualche decennio un pubblico estremamente composto, assistere alle partite addirittura senza barriere a separarlo dal campo da gioco (fatto per esempio ancora molto raro in Italia), a tutt'oggi il tifo d'oltremanica è spesso associato al terribile fenomeno degli hooligan. Impossibile dimenticare i numerosi episodi di violenza, culminati nella strage dell'Heysel del 1985. L'autore ne ricostruisce la nascita e l'evoluzione, a partire dagli epocali cambiamenti che il gioco del calcio ha vissuto in Inghilterra con l'inizio degli anni '80 dell'Ottocento.

\section{Premessa}

Il 29 maggio 1985, per la prima volta nella storia, la televisione documentò praticamente in presa diretta la tragica successione di eventi che si svolsero a margine della finale di Coppa dei campioni tra Juventus e Liverpool e che provocarono la morte di 39 persone, delle quali 32 italiani. Le cronache parlarono di scontri sanguinosi, della ressa, dei corpi calpestati e schiacciati a seguito del crollo del muro del settore Z. La strage dello stadio Heysel di Bruxelles mise di fronte agli occhi del mondo gli hooligan. Le dinamiche e i molteplici fattori che la provocarono sono stati più volte ricostruiti ed analizzati e non è questa la sede per analizzarli[1]. Tra questi ce n'è tuttavia uno che nello sconcerto e sbigottimento che accompagnò e seguì quella tragedia non fu colto 
immediatamente e che invece è indicativo della conoscenza approssimativa delle dinamiche e del fenomeno del tifo inglese. Questo fattore, che rende ancora più assurdo il bilancio finale di quella sera, sarebbe stato sottolineato qualche anno dopo dallo scrittore Nick Hornby nel suo romanzo autobiografico Febbre a 90' (ma il titolo originale era Pitch Fever) dedicato proprio alla passione calcistica e al tifo.

La causa di tutte quelle morti - scrive - fu qualcosa di tanto innocuo quanto una carica, esercizio che almeno la metà dei giovani tifosi inglesi praticava, e che non aveva altro scopo se non quello di spaventare i tifosi avversari e di divertire chi correva. I tifosi della Juventus, molti dei quali erano uomini e donne della media borghesia, non sapevano di questa abitudine, e d'altronde perché mai avrebbero dovuto? Non conoscevano il complicato comportamento del pubblico inglese, che noialtri avevamo assorbito quasi senza accorgercene. Quando videro una schiera di hooligan inglesi urlanti cominciare a correre verso di loro, si fecero prendere dal panico e si precipitarono verso l'estremità del loro settore. Crollò un muro, e nel caos che seguì la gente morì schiacciata. Fu un modo orribile di morire, e noi probabilmente davanti alla tv assistemmo a quelle morti: ricordiamo tutti quell'uomo robusto con la barba, assomigliante a Pavarotti, che con una mano cercava disperatamente un aiuto che nessuno riusciva a dargli.[2]

La carica (running) di cui parla Hornby era una delle tante "regole del disordine" - tra l'altro una delle più antiche perché ispirata ai rituali di supremazia territoriali in uso tra le gang dei giovani teppisti di strada di fine Ottocento-inizio Novecento (holding the street) - che vigevano da decenni negli stadi britannici[3]. Il fenomeno del calcio violento inglese era noto alle cronache ormai da anni ma pochi, soprattutto fuori dall'isola, ne conoscevano veramente le dinamiche.

\section{Il "football dei maestri" tra miti e stereotipi}

Occuparsi dello sport e del calcio in particolare, della sua pratica ma anche delle dinamiche e del senso di appartenenza del suo pubblico, significa studiare una delle «nuove tradizioni» che il processo di modernizzazione produsse o, per meglio dire (sempre per usare le parole di Eric J. Hobsbawm), "inventò" nel corso dell'Ottocento e del Novecento[4]. Il football moderno nasce e viene progressivamente codificato in Gran Bretagna trovando nella nascita della Football Association (1863) il suo evento fondativo. Per gli appassionati, ma anche per molti di coloro che scrivono e raccontano di questa disciplina, parlare del calcio britannico e di tutto ciò che ruota attorno ad esso significa dunque richiamarsi ad una tradizione circondata da un alone di mito che a sua volta, a seconda delle epoche storiche, si è rinnovata e modificata tingendosi di connotazioni ora positive, ora marcatamente negative. Non solo: alla "deformazione" - per usare uno degli attributi individuati da Barthes - che il mito opera sull'oggetto football si aggiunge la generalizzazione che è propria dello stereotipo, ovvero di una interpretazione che, in questo caso, rende "nazionali" caratteristiche o comportamenti di determinati gruppi o attori sociali[5].

Come molti studi sulle radici sociali del teppismo calcistico in Gran Bretagna hanno evidenziato, il rischio di una visione stereotipata del fenomeno è dunque sempre dietro l'angolo e, con esso, quello di incorrere in interpretazioni fuorvianti[6]. Gli esempi sono innumerevoli. Uno di questi è legato alla finale di FA Cup, il più antico torneo per club (inaugurato nel 1871), disputata a Londra il 25 marzo 1882 che vide prevalere per 1-0 la formazione degli Old Etonians, composta da ex studenti del prestigioso college del Berkshire, sulla squadra-simbolo della working-class delle regioni del nord, i Blackburn Rovers. 
La semplice lettura delle cronache dell'epoca di quella partita ribalta tanto l'archetipo dell'aristocratico praticante del football (spesso immaginato come il tipico gentleman vittoriano, dedito al fair-play e fedele al principio mens sana in corpore sano appreso nelle prestigiose public school del Regno) quanto quella, opposta, che vuole il giocatore di estrazione operaia rozzo e violento. In quel match, gli Etonians prevalsero infatti grazie ad un gioco ostruzionistico e brutale che rese vano il ricorso al più moderno gioco di passaggi che invece era prerogativa dei Rovers. Il calcio duro e violento dei rampolli dell'aristocrazia inglese del sud ebbe la meglio sul gioco manovrato degli operai del nord i quali, nonostante i duri scontri patiti, al fischio finale mostrarono anche un insospettabile «gentlemen spirit»[7]. Quella finale, nella quale il pubblico di entrambe le squadre si distinse per le grida di incitamento senza che però si registrassero incidenti, segnò il canto del cigno del "vecchio" football elitario. L'anno successivo, sempre a Londra, un altro club del centro industriale del Lancashire, il Blackburn Olympic, batté per 2-1 dopo i tempi supplementari proprio gli Old Etonians e la FA Cup venne così assegnata per la prima volta ad un club della classe operaia. Una storia, questa, che è stata a sua volta recentemente consegnata al grande pubblico, in chiave romanzata e con non poche inesattezze, nella serie televisiva per la piattaforma Netflix intitolata The English Game.

Ma in questo stesso tranello si rischia di incorrere anche e soprattutto nel momento in cui ci si propone di studiare, sia pure nei termini generali, come il pubblico calcistico agisce ma anche come viene rappresentato e di conseguenza percepito. Diversamente da quanto si potrebbe immaginare, solo una parte relativamente limitata del pubblico ha una conoscenza diretta del teppismo calcistico ma ne viene a conoscenza attraverso i media. Spiegare l'impatto che questi episodi hanno avuto nell'opinione pubblica e nell'immaginario collettivo aiuta dunque a spiegare anche le risposte fornite da parte degli attori politico-istituzionali in termini di provvedimenti concreti. Il modo con cui i media nel corso delle varie fasi storiche hanno presentato e commentato il tifo violento ha però influenzato non solo la percezione del pubblico in generale ma anche quella degli stessi tifosi, promuovendo o viceversa prevenendo effetti emulatori. Per questi e per molti altri fattori la tragedia dell'Heysel, che rappresenta un importante spartiacque per questo tema, rappresentò, tra le varie cose, anche una sorta di terribile profezia che si auto-avvera.

Per il caso britannico, una delle prime analisi che hanno cercato di indagare il fenomeno del tifo violento considerando anche la sua narrazione è quella fornita da Mason in uno dei capitoli centrali del suo studio sulle origini dell'Association football pubblicato nel 1980[8]. Questo lavoro metteva in evidenza la complessità di questa componente imprescindibile della pratica calcistica. Troppo spesso, infatti, come ha evidenziato Christian Bromberger, quando si tratta di studiare il pubblico «i folks beliefs (“credenze popolari”) e gli stereotipi» tendono a sostituirsi «all'informazione». Di fronte a queste letture semplicistiche o affrettate, il pubblico diventa quindi un «oggetto paradossalmente sconosciuto»[9].

Il tifo inglese non sfugge a tutto ciò. Anche su questo versante miti e stereotipi si intrecciano. Almeno fino alla metà degli anni Sessanta, ad esempio, tanto nel Regno Unito quanto tra molti osservatori esterni prevaleva (sulla base di una visione stereotipata del "carattere nazionale" britannico) una immagine del pubblico inglese come di un pubblico tradizionalmente sportivo e civile, capace di sostenere calorosamente la propria squadra e di accettare sportivamente l'esito del campo, qualsiasi questo fosse. Questa concezione, pur non scomparendo del tutto neppure successivamente, è stata poi sostituita da quella diametralmente opposta, che vedeva invece il tifo inglese incarnato dal fenomeno degli hooligan. Proprio sul tifo violento inglese sono incentrati molti degli studi più approfonditi anche se, soprattutto inizialmente, di taglio prevalentemente sociologico. Cedere a certe visioni standardizzate è però doppiamente pericoloso: innanzitutto perché, ma questo è facile intuirlo, il tifo inglese non è stato soltanto hooliganism ma qualcosa di ben più complesso ed articolato. Inoltre, ricostruzioni storiche di più lungo periodo mostrano come 
in realtà la violenza legata al football britannico non appartenga solo alla storia novecentesca ma abbia in realtà radici ben più antiche che dunque ne mettono in discussione proprio il mito "positivo". La «comunità immaginata» del tifo, che presenta molti tratti in comune alle altre "comunità immaginate" di Benedict Anderson, a cominciare dal senso di "profondo, orizzontale cameratismo» è dunque un soggetto in costante trasformazione sul quale il dibattito è ancora aperto[10].

\section{Nasce il "gioco del popolo"}

Come alcuni dei primi studi sulle origini del movimento calcistico britannico hanno messo in evidenza, il football passò da un gioco riservato alla upper e middle class ad uno sport con una base sociale sempre più ampia, assumendo caratteristiche molto vicine a quelle del calcio moderno, nell'arco di un brevissimo lasso di tempo[11]. Il mutamento avvenne durante i trenta-quaranta anni che seguirono l'inizio del passaggio al professionismo avvenuto a partire dagli anni Ottanta dell'Ottocento e di cui la finale di FA Cup vinta dal Blackburn Olympic e la nascita della Football League (1888) furono due importanti momenti di svolta. Già nel primo Novecento, insomma, la "febbre del football" era esplosa, come confermano i dati relativi alle decine di migliaia di spettatori che mediamente seguivano le fasi finale della FA Cup o le partite della First Division[12]. Ma qual era la composizione sociale del pubblico che seguiva questa prima fase di sviluppo del movimento britannico? La letteratura che dagli anni Settanta in poi si è occupata di questo aspetto ha messo in relazione il significativo incremento della media-spettatori con la progressiva riduzione dell'orario di lavoro per alcuni settori dell'industria il sabato. L"'invenzione del week-end", frutto della legislazione di fabbrica, contribuì a fare degli operai dell'industria pesante una componente sempre più rilevante del pubblico calcistico. Ad essi si aggiunsero gradualmente anche altri lavoratori manuali qualificati ma anche membri del piccolo ceto impiegatizio e del ceto medio. Gli elementi del sottoproletariato o comunque la rough working class, anche per una precisa politica dei prezzi dei biglietti di ingresso, erano una minoranza. Per quanto in maggioranza di estrazione operaia e popolare, dunque, la "folla" calcistica di fine secolo mostrava una composizione variegata sia per estrazione, che per classi di età e persino per genere (e ciò in maniera ancor più marcata fuori dalla capitale e in realtà come la Scozia).

Le cose cominciarono a cambiare nel corso degli anni Novanta dell'Ottocento quando la divaricazione tra calcio professionistico di vertice e calcio dilettantistico andò accentuandosi. Un insieme di fattori tra i quali l'aumento del pubblico, la crescente scomodità degli impianti - molti dei quali, a conferma del legame sempre più stretto del gioco con la classe operaia sorgevano a ridosso delle aree industriali o in mezzo alle case - ma anche gli incidenti tra tifosi contribuirono probabilmente ad allontanare dallo spettacolo calcistico dei club professionistici le donne e crescenti settori dei ceti medi e di quelli medio-alti. Dallo spoglio della stampa nazionale e locale contenute sin dalle prime ricerche degli anni Ottanta, in particolare quelle del Centro di Leicester sul teppismo calcistico in Inghilterra, emerge infatti che già dagli anni '70 dell'Ottocento i disordini e le violenze legate alle partite di calcio crescono sul piano numerico[13].

Il modo con il quale il pubblico sosteneva la propria squadra era ancora relativamente disorganizzato: non esistevano cori o canti veri e propri né sciarpe con i colori della squadra. Quali erano le più comuni intemperanze dei tifosi? L'invasione del campo, l'aggressione all'arbitro o alla squadra ospite, il lancio di oggetti, episodi di vandalismo anche gravi, risse con feriti erano gli episodi più frequenti. Fatti spesso anche gravi che in taluni casi richiedevano l'intervento di decine di agenti polizia e persino dei vigili del fuoco armati di autopompe. Gli incidenti dell'aprile 1889 tra i sostenitori del Nantwich e del Crewe, avvenuti all'interno della stazione ferroviaria di Middlewich 
gremita di passeggeri, o come quelli tra Morton e Port Glasgow di dieci anni dopo, con scontri che dilagarono per le vie cittadine obbligando i negozianti a chiudere le loro attività per evitare vandalismi, sono emblematici di una violenza che già in questa fase non era più confinata al terreno o agli impianti di gioco[14].

La stampa dell'epoca, come detto, si rivela la fonte privilegiata (insieme alle carte e ai verbali della Football Association) per analizzare tipologia, incidenza ma anche percezione del "tifo violento" da parte del pubblico anche in questa fase. Va infatti sottolineato come l'ascesa del football avvenne in contemporanea - e sotto molti aspetti fu fortemente favorita - dalla straordinaria crescita della stampa in generale e di quella sportiva in particolare, una informazione sempre più «fattuale e rapida» che riusciva ad aggiornare i lettori con più edizioni giornaliere, in taluni casi addirittura quattro in due ore[15]. La diffusione dell'ubriachezza, gli atteggiamenti e il linguaggio aggressivo del pubblico, i disordini associati alla football madness (come la definì Ernest Ensor nel 1898), il diffondersi del professionismo e persino i mutamenti dello stile di gioco (paradossalmente, la sua spettacolarizzazione, giudicata eccessiva) furono spesso al centro di articoli e editoriali, della stampa che si rivolgeva a lettori delle upper e middle class. In alcuni casi, il biasimo era basato su questioni fondate: i controlli agli ingressi erano praticamente inesistenti e sulle terraces si beveva molto, con ciò che spesso ne conseguiva[16]. Tuttavia, dietro queste critiche, che spesso si accompagnavano alla nostalgia per quello che era stato il passatempo invernale delle classi elitarie, spesso c'era una più generale e preconcetta riprovazione da parte dei ceti più abbienti nei confronti della classe lavoratrice. Il fatto che questo fenomeno si sovrapponesse sul piano temporale con quello delle bande giovanili degli emuli della famigerata Hooley's Gang che operava nell'East End londinese, gli hooligan, non è un elemento da sottovalutare[17]. In un contesto che pure tollerava un certo livello di violenza, il comportamento riprovevole di una parte degli spettatori - definiti con epiteti quali «animali», «folli» (lunatics), «teppisti» (thugs) - rappresentava secondo alcuni osservatori della tarda età vittoriana e di quella edoardiana, una conferma indiretta della «scarsa rispettabilità» e della «aggressività» di buona parte della classe operaia[18]. In altri termini, anche il calcio professionistico e la football madmess finiva per essere oggetto di riprovazione al pari di altre espressioni della cultura popolare come il teatro di varietà o i romanzi a puntate dai contenuti giudicati violenti o immorali come i penny dreadful[19]. Sul versante diametralmente opposto erano attestati, per ovvie ragioni, i dirigenti della Football Association e della Football League che invece «non smettevano di declamare che il football aveva effetti moralizzatori e che le partite riunivano le frazioni più rispettabili della working class»[20].

\section{Flemma e fair-play: un mito che si autoalimenta}

Al momento dello scoppio della Grande Guerra, il calcio in Inghilterra aveva dunque ormai assunto il carattere di "gioco del popolo" (people's game)[21]. La squadra, lo stadio, i suoi sostenitori, e ogni altro aspetto che ruotava attorno al rito settimanale del match costituivano altrettanti tratti distintivi di uno sport che era appannaggio quasi esclusivo della classe operaia e da essa considerato parte integrante della propria cultura. Il fenomeno stava già assumendo una dimensione europea come dimostrava indirettamente l'episodio, anch'esso avvolto dall'alone del mito, della breve tregua di Natale del 1914 secondo il quale i soldati inglesi e tedeschi avevano fraternizzato improvvisando una partita di calcio nella terra di nessuno in mezzo alle trincee[22]. Quella del 1914-15 fu l'ultima stagione del massimo campionato prima della interruzione forzata, accompagnata da un dibattito appassionato tra favorevoli e contrari alla prosecuzione dei campionati, dovuta proprio al conflitto[23]. 
Il periodo tra le due guerre si rivela decisivo proprio ai fini della nascita e del radicamento del mito (positivo) della correttezza del pubblico calcistico britannico. I dati di questi anni relativi ai disordini e ai provvedimenti disciplinari adottati dalla Federazione risultano stabili o (ad esempio per il caso scozzese) addirittura in aumento ma la gravità degli episodi sembra notevolmente ridimensionarsi e, con essa, quella delle sanzioni comminate. Ciò non significa che il comportamento antisportivo o violenta del pubblico - che continuò ad aumentare sul piano numerico a testimonianza della ulteriore crescita del movimento - non trovasse spazio sui giornali. Come hanno evidenziato Eric Dunning, Partick Murphy e John Williams

È probabile che l'aumento della frequenza con cui, tra le due guerre, vengono riportate sulla stampa le condotte "non sportive" e di "scherno" sia una conseguenza del fatto che allora ci si aspettava dalle folle calcistiche un comportamento più elevato sotto tutti gli aspetti, per cui le infrazioni comportamentali relativamente meno gravi risaltano in modo più netto di prima. Se così stanno le cose, questo andamento sarebbe coerente con una delle tesi di Durkheim sul crimine: quando l'incidenza di una forma particolare di comportamento criminale diminuisce, altre forme di condotta deviante, o socialmente inaccettabile, fino ad allor considerate meno gravi, iniziano ad essere considerate altrettanto biasimevoli dei crimini in diminuzione.[24]

Per quanto segnalati dalla stampa, gli episodi negativi vengono in qualche modo messi in secondo piano, vivono in una sorta di piano di esistenza parallelo e sotterraneo nel quale invece sembra prevalere una rappresentazione del tifo positiva, in linea con lo stereotipo (positivo) del carattere nazionale. Si tratta di una «immagine di civilizzazione», come è stata definita da Michael Eve, che si nutre di una specifica «gamma di rappresentazioni associate allo status di una nazione»: nel corso dei secoli l'immagine dei britannici - così come quella di altri abitanti di paesi considerati più "moderni" e "civilizzati" - è stata associata ad attributi quali «disciplinato», «emotivamente controllato», «formale» ecc[25]. Dietro questo meccanismo si celava anche, per usare le parole di Norbert Elias, la volontà da parte di molti mezzi d'informazione di diffondere, all'interno e all'esterno del paese, l'immagine di una classe operaia pienamente integrata «negli standard di consuetudini e di comportamenti che la società cercava [...] di imporre ai singoli»[26].

Mentre dunque si costruiva e consolidava il mito della flemma e sportività del pubblico britannico, facendo peraltro riferimento ad una tradizione che in realtà - come abbiamo visto - era "inventata", il «culto proletario di massa»[27] conservava anche la sua connotazione violenta ma questa, diversamente dalla fase precedente, restava celata, sottotraccia.

La finale di FA Cup del 1923 tra West Ham United e Bolton Wanderers giocata, come recitava la didascalia in apertura del videogiornale della Pathé Film, nel «nuovo, meraviglioso stadio di Wembley» diventò dunque il simbolo della compostezza e della maturità del tifoso inglese. In quella occasione, una folla immensa, calcolata tra le 250.000 e le 300.000 persone, raggiunse lo stadio (che ne poteva contenere solo 125.000) riempiendolo in ogni ordine di posti e assiepandosi fino a bordo campo[28]. La questione giunse in Parlamento, dove il ministro dell'Interno finì sul banco degli imputati perché, come emerse dal dibattito, almeno 1.000 persone erano rimaste ferite[29]. Eppure, nonostante ciò, la maggior parte dei resoconti evidenziò non tanto la pericolosità dell'episodio e il fatto che la tragedia era stata evitata per un soffio quanto piuttosto l'entusiasmo, tra il pittoresco e il patologico, con il quale la marea di tifosi aveva riempito l'Empire Stadium («il più grande stadio del mondo») seguendo il match con straordinaria compostezza e persino, come scrisse il Times, con «amazing good humour and patience»[30].

Le immagini della White Horse Final del '23 (così chiamata in omaggio a Billy, il cavallo montato da uno dei poliziotti chiamato a gestire l'ordine pubblico, che spiccò in molte fotografie di quel giorno in mezzo al grigio formicaio umano degli spettatori bianco - ma in realtà il suo manto era 
grigio chiaro) dicono anche altro, ovvero la netta preponderanza di un pubblico maschile. Tuttavia, evidentemente per il fatto che gli stadi di calcio erano tornati ad essere percepiti come luoghi più sicuri rispetto al passato, proprio in questo periodo la partecipazione di pubblico femminile, persino privo di accompagnatori maschili, aumentò sensibilmente[31].

\section{Il calcio va alla guerra?}

Una visione altrettanto consolidata dell'evoluzione del tifo inglese nel secondo dopoguerra, avvalorata in molti casi da articoli e inchieste giornalistiche, vuole che l'inizio della spirale di violenze destinata a culminare con il fenomeno degli hooligans abbia inizio con la seconda metà degli anni Sessanta[32]. Questa periodizzazione trae spunto da almeno un paio di fattori. Il primo è legato al fatto che gli anni che seguirono la fine del secondo conflitto mondiale rappresentarono l'apogeo del mito positivo del pubblico inglese. Almeno fino alla prima metà degli anni Cinquanta, il numero di incidenti registrati rimase basso, soprattutto se messo in relazione all'altissima affluenza di spettatori. Tutto questo contribuì a rafforzare l'immagine del calcio come "gioco del popolo" e quella dei suoi principali attori, i calciatori, come "eroi della classe operaia"[33]. La vittoria ai mondiali del 1966, letta come il trionfo della scuola britannica, costituisce l'altro fattore che ha rafforzato questa interpretazione. In realtà, ancora una volta, una lettura meno schematica fornisce indicazioni di segno diverso.

In realtà l'immagine del football come gioco per i settori "rispettabili" della classe operaia cominciò a declinare già nel corso degli anni Cinquanta, in concomitanza con l'emergere delle prime subculture giovanili che, proprio a partire dalla metà di quel decennio finirono sotto i riflettori dei mezzi di comunicazione, in particolare della stampa popolare scandalistica. Il primo nesso tra fenomeni di "panico morale" nei riguardi di questi gruppi di giovani e la violenza negli stadi coincise con il diffondersi dei Teddy Boy. La ribellione nichilista delle bande composte da questi giovani proletari vestiti in abiti edoardiani, amanti del rock and roll e della violenza, che, dal delitto di Clapham Common del luglio del 1953 ai disordini razziali di Nottingham e di Notting Hill del 1958, furono al centro delle cronache, si mescolò alle notizie che riportavano la ripresa delle violenze dentro e fuori gli stadi. Il tentativo di usare proprio gli eroi del calcio come testimonial di campagne moralizzatrici (fu questo il caso di Stanley Matthews che ricevette questo compito addirittura su iniziativa del Duca di Edimburgo) andò sostanzialmente fallito: il fenomeno dei Ted varcò il canale della Manica per diventare una delle chiavi di lettura del dibattito in corso in questi anni proprio sulla devianza e il ribellismo giovanile[34].

Questa maggiore sensibilità nei riguardi delle violenze nascondeva però, secondo altre interpretazioni, anche altre cause. Di fronte allo shock provocato da alcune pesanti sconfitte della nazionale (il "miracolo di Belo Horizonte" legato alla clamorosa eliminazione della nazionale inglese a opera degli Stati Uniti ai mondiali del 1950 e il Match of the Century del 1953 che vide i bianchi d'Inghilterra sconfitti sonoramente a Wembley dalla nazionale ungherese) e ad un calo degli spettatori si stava facendo strada l'idea di trasformare il calcio in uno sport più adatto alle famiglie e più in generale capace di attrarre un pubblico diverso da quello di estrazione operaia. Gli anni Sessanta si aprirono infatti all'insegna di una prima modernizzazione del football[35].

Nel corso della prima metà degli anni Sessanta alcuni episodi di violenza legati al calcio, commessi in particolare da giovanissimi tifosi posero l'attenzione nuovamente sul problema della devianza minorile e della "nuove" bande di giovani ribelli. La schoolboy threat si andò quindi saldando, com'era del resto avvenuto per i Teddy Boy, con l'ondata di panico morale che si accese nei riguardi dei Mod e dei Rocker all'indomani degli scontri e atti di vandalismo registratisi in alcune 
località di villeggiatura del Sud dell'Inghilterra proprio tra il 1964 e il 1965[36]. Il "vecchio" mito della sportività, flemma e correttezza dello spettatore inglese, che pure era stato rispolverato da una parte della stampa britannica all'indomani degli incidenti che si verificarono allo stadio Flaminio in occasione della partita di Coppa delle Fiere tra Roma e Chelsea e messo in contrapposizione alle violenze e intimidazioni dei tifosi latini stava tramontando per sempre. Pochi giorni dopo disordini e scontri funestarono anche le gare del campionato inglese e si ripeterono nelle settimane e nei mesi successivi a conferma che l'Inghilterra non era affatto immune da quella che era definita una «malattia» universale[37]. Il sensazionalismo della stampa giocò un ruolo decisivo, amplificando fenomeni che certo erano un pericoloso campanello d'allarme ma che nello stesso tempo, proprio per questa crescente esposizione mediatica, finirono spesso per autoalimentarsi, entrare nell'immaginario collettivo e poi diventare un vero e proprio modello. I tifosi del calcio erano divenuti i nuovi folk devils. Uno degli episodi-chiave in questo senso avvenne nel novembre del 1965, quando durante Brentford-Millwall, dal settore dei Lions venne lanciata in campo una bomba a mano priva di innesco. Il "giorno della bomba" fu commentato dal Sun con un titolo emblematico: Soccer Marches to War!

Nel corso degli anni Sessanta, complice il diffondersi delle subculture giovanili, all'interno degli stadi inglesi (o per meglio dire nelle varie End, cioè i settori più caldi) era emersa e si era progressivamente definita ed affermata una specifica subcultura con ritualità e "mentalità" proprie e precise «regole del disordine»[38]. Alcune tifoserie hanno particolarmente contribuito in tal senso: si pensi al ruolo della cultura scouse e scally nella Liverpool degli anni Sessanta[39]. Tradizione popolare, alcuni elementi della cultura pop (musica, mode e stili), comportamenti delle bande di quartiere si fusero insieme e contribuirono alla creazione di questa precisa identità del "nuovo" tifoso di calcio. L'emergere del fenomeno degli Skinhead, alla fine del decennio, rappresentò un ulteriore detonatore.

\section{L'emergere della "malattia inglese": tra panico morale e subculture giovanili}

A partire dalla seconda metà degli anni Sessanta il movimento dei Mod si divise in diversi rivoli. Da uno di questi emersero i primi gruppi di giovani portatori di un proprio nuovo stile. Portavano i capelli rasati, simili a quelli dei soldati e degli astronauti americani visti in televisione o al cinema, e ciò indusse il Daily Mirror a chiamarli con l'appellativo Skinhead. La diffusione su larga scala di questa nuova subcultura giovanile coincise con la perdita della originaria connotazione multiculturale ed accentuò viceversa un carattere spiccatamente violento e xenofobo:

Capelli rasati, scarponi da lavoro, jeans con bretelle, "nervosismo paranoico": tra il 1967 e il 1969 gli hard mod assumono il nome di skinheads, esprimendo tutte le caratteristiche di una sottocultura originale, basata sulla passione per il football e sulla rielaborazione in chiave working class degli atteggiamenti e dei consumi musicali della micro-delinquenza giovanile giamaicana.[40]

Nel clima di crescente malessere che contraddistinse il Regno Unito sin dai primi anni Settanta, le preoccupazioni per la crisi economica, l'aumento della criminalità, di fenomeni come il razzismo e la xenofobia (si pensi alla diffusione del National Front) fecero sì che attorno al fenomeno degli skinhead, sempre più presente sugli spalti, si scatenasse una nuova e stavolta ben più rilevante ondata di panico morale. Gli incidenti della primavera del 1970, simili a quelli tra i Mod e i Rocker, e gli altri episodi di violenza dei gruppi di Skin ancora una volta si saldarono con il moltiplicarsi delle notizie che riferivano di scontri, risse, incidenti ed arresti dentro e fuori gli stadi. È in questo contesto che si andarono ulteriormente definendo le modalità di confronto-scontro tra i diversi 
gruppi di tifosi che sceglievano la via della violenza come estrema modalità di contrapposizione a tutto ciò che rifiutavano ma anche, più banalmente, per semplice divertimento. Tutto questo in un contesto sociale che vedeva la classe operaia tradizionale (e la sua cultura) erosa da un lato da un processo di imborghesimento e dall'altro da un processo di marginalizzazione che il Daily Mirror sintetizzò nella espressione Aggro Britain ${ }^{[41]}$. Gli stadi erano diventati dei luoghi pericolosi sia per le violenze scaturite dal diffondersi della cultura aggro ma anche perché erano infrastrutture vecchie e fatiscenti. Le 66 vittime e le centinaia di feriti provocati dalla calca di Ibrox Park del gennaio del 1971 rappresentarono in tal senso una tragica presa d'atto del dissesto in cui versavano molti impianti.

Le interpretazioni della crisi del calcio si moltiplicarono: l'occasione di fare della vittoria storica del 1966 una occasione per portare a termine la modernizzazione del football era chiaramente fallita. Il livello tecnico-spettacolare si era abbassato. Persino i giocatori, con la loro mancanza di fair-play e i loro atteggiamenti isterici o violenti, contribuivano a fomentare le folle. Qualche giornale si affrettò a ribattezzare il campionato come la Thugs League, il campionato dei teppisti[42]. Si invocarono sanzioni più dure in campo e fuori senza che però ottenere risultati. L'eliminazione della nazionale inglese dalla fase finale dei mondiali ad opera della Polonia, nell'ottobre del 1973, fu colta come un ulteriore segnale di declino. Il colpo finale giunse l'anno successivo quando ai gravi incidenti verificatisi a Rotterdam in occasione della finale di ritorno della Coppa Uefa tra il Feyenoord e il Tottenhan Hotspur - che portarono agli occhi del mondo l'esistenza della "malattia del tifo inglese" - si aggiunse la morte per accoltellamento di un diciassettenne durante la partita del campionato di seconda divisione tra Blackpool and Bolton per mano di un tifoso avversario quattordicenne. La "battaglia di Rotterdam", come venne definita dalla stampa con il ricorso ad un linguaggio bellico che agli occhi di molti finì per trasformare i tifosi violenti in moderni guerrieri[43], fu importante spartiacque. A poco valevano gli epiteti - divenuti una consuetudine con i quali vennero definiti i tifosi violenti («animals», «thugs», «disgraceful» «bad losing brawlers»): l'indiretta spettacolarizzazione della violenza e in certi casi la visibilità mediatica fornita ad alcuni dei protagonisti egli scontri contribuì a fissare nell'immaginario collettivo britannico e più in generale europeo il fenomeno del football hooliganism.

A partire dalla seconda metà degli anni Settanta, con la formazione e le imprese delle varie Crew (si pensi alla Red Army del Manchester United e al suo slogan We hate humans!), ciascuna con precise rivalità ed alleanze e modus operandi codificati - a cominciare dal già citato rito della "conquista della tribuna" avversaria (holding the End) - l'hooliganism era diventato - complici le competizioni calcistiche europee e la televisione - una sorta di modello, ammirato ed in certi casi imitato dalle tifoserie violente di mezza Europa. Già alla fine degli anni Settanta gli hooligan erano diventati i nuovi folk devils[44]. Lo stile si era nel frattempo evoluto: gli Skinhead, per i quali la violenza e il rito calcistico del sabato rappresentava un modo di difendere inconsciamente una cultura proletaria che andava scomparendo o ghettizzandosi, stavano per lasciare spazio ai Casual che, con il loro abbigliamento firmato e persino con le loro "tecniche di ingaggio", rappresentavano paradossalmente, sia pure in modo inconsapevole, il segnale dell'imborghesimento anche del tifo violento.

\section{Dai Superhooligan al tifo da poltrona}

Nel corso degli anni Ottanta il tifo violento inglese raggiunse il suo apice. Quali che fossero le cause di questo fenomeno - la reazione dei tifosi working class nei confronti dell' imborghesimento del calcio, l'erosione della classe operaia provocata crisi dell'industria o il rifiuto del processo di incivilimento della società - esso mostrò di aver mutato nuovamente pelle. La tradizionale figura 
dell'hooligan consolidatasi nei decenni precedenti stava infatti lasciando spazio ad un nuovo idealtipo di tifoso violento. La strage dell'Heysel, trasmessa in diretta televisiva in tutta Europa (solo la rete nazionale tedesca ZDF, una volta emersa la portata degli incidenti, decise di interrompere la messa in onda), ebbe l'effetto di porre definitivamente all'attenzione generale questa nuova mutazione. I termini del dibattito che seguirono quei tragici eventi non erano molto diversi da quelli che già avevano caratterizzato i mesi e gli anni precedenti. Alla condanna unanime e ai consueti epiteti rivolti agli hooligan («selvaggi», «belve impazzite», «barbari», «fanatici», «sciacalli») si accompagnò però la presa di coscienza che l'identikit del teppista di stadio inglese era cambiato. Come scrisse l'Observer in un editoriale:

«the modern football hooligan is not a glue-sniffing skinhead in Dr. Marten's boots. He is a Cortina or capri-driving employed follower of high fashion, and he as proud of his ability to afford Armani, Woodhouse and Nickleby as he is ready to slash your face with a Stanley knife before you can slash his» ${ }^{[45]}$

Il profilo, dunque, non corrispondeva affatto, né sul piano generazionale né su quello della condizione sociale, con il classico tifoso violento del passato. Si era in presenza di persone adulte, mediamente istruite, appartenenti al ceto medio, con un profilo occupazionale che andava dal lavoratore semiqualificato al professionista. Vestivano con costosi capi firmati (Burberrys', Pringle, Sergio Tacchini, Fila, Fred Perry, Lacoste, Ellesse, Church, Ben Sherman, Lyle \& Scott), si spostavano in gruppi di alcune centinaia di elementi, adottavano tecniche elusive nei confronti della polizia evitando i treni speciali di tifosi, preferendo viceversa i treni più costosi come gli Intercity e avevano disponibilità economiche che permettevano loro di seguire le loro squadre anche $\mathrm{e}$ soprattutto fuori dai confini nazionali. Questi gruppi di superhooligan, come furono presto ribattezzati, erano dotati di una propria gerarchia interna e di leader riconosciuti[46].

Ancora una volta, tuttavia, il quadro restava assai più complesso di quanto appariva da molte inchieste. Il "modello" del teppista-tifoso inglese mutava «in relazione con le trasformazioni conosciute dalla classe operaia, da una specifica generazione, e in risposta alle nuove condizioni imposte dalle autorità» ma nel contempo manteneva alcuni tratti del passato. Oltre ad un forte senso del gruppo e di appartenenza (un "noi" contrapposto ai "loro") queste costanti comprendevano lo «stile mascolino aggressivo», il senso di cameratismo, la ricerca di emozioni forti in un contesto sociale viceversa contraddistinto dalla depressione e dalla ripetitività, la «sospensione del normale ordine delle cose» che si accompagnava al rituale della partita o all'esperienza della trasferta, il farsi beffe della «società civilizzata» abbandonando autocontrollo o ricorrendo a volgarità o frasi di scherno moralmente riprovevoli (i cori contro i tifosi o i giocatori avversari scomparsi ecc.)[47].

Il superhooligan era però anche e soprattutto un prodotto mediatico che la stampa popolare, i tabloid in particolare, avevano contribuito a costruire e diffondere con titoli iperbolici, cronache sensazionalistiche e servizi dedicati ai principali leader[48]. Anche se non contribuisce a spiegare del tutto il fenomeno, l'interazione tra media e stampa, con la conseguente "costruzione della notorietà» degli hooligan, è un aspetto centrale di questa fase della storia del tifo violento britannico[49]. Le cronache contribuirono ad accrescere il mito delle varie fighting crew e dei loro capi: la Inter City Firm del West Ham, i Bushwackers del Millwall, i Gooners dell'Arsenal, la Baby Squad del Leicester, gli Headhunters del Chelsea, la Service Crew del Leeds United.

Banalizzazioni, interpretazioni stereotipate o artefatte allo scopo di impressionare il pubblico fanno parte di questa particolare modalità narrativa del fenomeno e della sua diffusione anche fuori dai confini britannici. Un esempio in tal senso è in parte rappresentato dal libro di Bill Buford nel quale egli ricostruiva da infiltrato, anche in questo caso con intenti sensazionalistici, le imprese di alcuni gruppi di hooligan. Il libro uscì in Italia con un titolo I furiosi della domenica (mentre, com'è noto, in Inghilterra si giocava prevalentemente di sabato), con un sottotitolo altrettanto fuorviante (che 
assimilava due differenti tipologie di tifo estremo) Viaggio al centro della violenza ultrà e con una copertina che ritraeva una carica della polizia italiana nei confronti degli ultras della Fiorentina[50].

Sempre nel corso degli anni Ottanta, eventi tragici come il rogo del Valley Parade di Bradford, avvenuto pochi giorni prima della strage dell'Heysel e costato la vita a 56 spettatori, e la strage di Hillsborough a Sheffield, nella quale morirono 96 tifosi del Liverpool, schiacciati dalle recinzioni erette proprio per evitare incidenti tra tifosi, accelerarono il processo di un cambiamento che in parte era già in corso. Ai severi provvedimenti restrittivi decisi dal governo Thatcher e applicati dalle forze di polizia anche con il ricorso a tecniche di infiltrazione simili a quelle usate per contrastare il terrorismo interno[51], si aggiunsero delle misure improntate alla salvaguardia della sicurezza degli spettatori negli stadi. Le conseguenze finanziarie di queste scelte sull'intero movimento calcistico inglese, posto ormai sul banco degli imputati dai media e dell'opinione pubblica, furono pesanti ed ebbero delle serie ripercussioni per le casse di molti club[52]. I mondiali di Italia '90 segnarono a loro volta un ulteriore ma stavolta positivo momento di svolta. La nazionale inglese non vinse quella competizione ma vinse il trofeo Fair Play della FIFA e i temuti tifosi inglesi ebbero un comportamento tutto sommato accettabile. Il pubblico si riconciliò con i suoi beniamini in un clima di ritrovato patriottismo nostalgico riavvicinamento al "gioco del popolo" che Nick Hornby avrebbe poco dopo fissato nel romanzo autobiografico Fever Pitch.

Fu in questo contesto che si giunse alla nascita della Premier League. La Premier fu un ulteriore e decisivo passo in direzione della modernizzazione del calcio inglese. La finanziarizzazione del football e l'irrompere della televisione modificarono in modo indelebile anche il modo di assistere allo spettacolo calcistico. La costruzione di stadi all-seated, cioè con solo posti a sedere, segnò la fine delle terrace e di un modo di fare tifo, sancendo l'avvento del tifoso cliente/consumatore ${ }^{[53]}$. Già nel 1993 uno studio sul pubblico dell'Aston Villa e dell'Arsenal evidenziava come la metà degli spettatori della squadra di Birmingham e addirittura i due terzi di quella londinese erano «impiegati o liberi professionisti» e comunque appartenevano alle classi medie[54].

Mentre dunque il fenomeno dell'hooliganism, colpito al cuore dalla repressione ma non eradicato del tutto, andò in parte declinando (gli scontri, ad esempio, si spostarono sempre più fuori e spesso addirittura lontano dagli stadi), una parte del tifo sentendosi marginalizzata e demonizzata dall'opinione pubblica e contraria a questa svolta cercò di opporsi al cambiamento. Si trattava di gruppi di supporter indipendenti non legati alle storiche associazioni riconosciute e che restavano fedeli all'idea del calcio come uno sport per la classe operaia rifiutandone l'imborghesimento provocato dal crescente costo dei biglietti e la perdita dei legami con la comunità locale/nazionale. Proprio attorno alla difesa degli spazi identitari (in primis gli stadi) e in alcuni casi della sopravvivenza stessa della propria squadra in questa fase si mobilitarono pacificamente i tifosi del Charlton Athletic, del Wimbledon, del Bournemouth, il Brighton e poi quelli del West Ham, del Manchester United del Manchester City, del Norwich, del Reading e del Watford. I tifosi stampavano pubblicazioni indipendenti (fanzine), autofinanziate e autoprodotte (The City Gent, The Gooner, Voice of the Valley, Fly Me to the Moon, The Oatcake, United We Stand ecc.) e si organizzavano in una rete destinata a crescere nel corso degli anni. Era l'emergere di un originale e forma di football activism contrario al tifo da poltrona che contribuì a rivalutare l'immagine del tifoso di calcio, schiacciata fino a quel momento sullo stereotipo dell'hooligan. Un approccio critico ai primi segnali dell'avvento del calcio globalizzato che trova oggi un suo parziale richiamo nel movimento che, assunta una dimensione sovranazionale, si unisce sotto lo slogan Against Modern Football ${ }^{[55]}$. Non solo hooliganism, dunque. Il «terzo secolo del football», con le sue trasformazioni, era iniziato[56]. 
Note:

[1] Si veda, ad esempio, S. Govaert, M. Comeron, Foot \& Violence, Politique, stades et hooligans. Heysel 85, , Université De Boeck Bruxelles 1995.

[2] N. Hornby, Febbre a 90’, Guanda, Parma 1997 [1992], p. 153.

[3] Cfr. G. Pearson, Hooligan. A History of Respectable Fears, MacMillanl, LondonBasingstoke1983, p. 84.

[4] E.J. Hobsbawm, Introduzione: Come si inventa una tradizione, in E.J. Hobsbawm e T. Ranger (a cura di), L'invenzione della tradizione, Einaudi, Torino 2002 [1983], p. 13.

[5] Cfr. R. Barthes, Miti d'oggi, Einaudi, Torino 2016 [1957] in particolare pp.203-209.

[6] E. Dunning, P. Murphy, J. Williams, Teppismo calcistico in Gran Bretagna: 1880-1889, in A. Roversi, Calcio e violenza in Europa. Inghilterra, Germania, Italia, Olanda, Belgio e Danimarca, Il Mulino, Bologna 1990, p. 52.

[7] D. Steel, N. Soames, The Essential Englishman. An Anthology, Cassel, London 1989, pp. 172173.

[81 T. Mason, Association Football and English Society, Harvester, Brighton 1980, pp. 138-174.

[9] C. Bromberger, La partita di calcio. Etnologia di una passione, Editori Riuniti, Roma 1999 [1995], p. 20.

[10] B. Anderson, Comunità immaginate. Origini e fortuna dei nazionalismi, Laterza, Bari-Roma 2018 [1983, 1991], pp. 11-12.

[11] E. Dunning, P. Murphy, J. Williams, The Roots of Football Hooliganism. An Historical and Sociological Study, Routledge \& Kegan Paul, London-New York 1988, pp. 32 e ss.

[12] Mason,1980, pp. 141-143.

[13] Sulla "Scuola di Leicester" si veda ad esempio: A. Bairner, The Leicester School and the Study of Football Hooliganism, in "Sport in Society", Volume 9, 2006, Issue 4, pp. 583-598 e S. Best, The Leicester School of Football Hooliganism: an evaluation, in "Soccer and Society", Volume 11, 2010, Issue 5, pp. 573-587.

[14] Dunning, Murphy, Williams, 1990, pp. 26-29.

[15] Cfr. R. Colls, This Sporting Life. Sport \& Liberty in England, 1760-1960, Oxford University Press, London-New York 2020, p. 261.

[16] Si veda ad esempio J. Spurling, Highbury. The Story of Arsenal in N. 5, Orion, London 2006, p. 19. 
[17] Cfr. V. Marchi, Ultrà. Le sottoculture giovanili negli stadi d'Europa, Hellnation, Roma 2015, pp. 26-30.

[18] Cfr. Dunning, Murphy, Williams, 1988, pp. 76-90.

[19] Pearson, 1983, pp. 63 e ss.

[20] P. Dietschy, Storia del calcio, Paginauno Mc’Nelly, Vedano al Lambro 2010, p. 57.

[21] R. Holt, La tradition ouvriériste du football anglaise, in "Actes de la recherche en sciences sociales", Vol. 103, Juin 1994, p. 38.

[22] Cfr. I. Adams, A Game for Christmas? The Argylls, Saxons and Football on the Western Front, December 1914, in "The International Journal of the History of Sport", 32 (11-12), 2015, pp. 1395-1415; M. Brown, The Christmas Truce 1914: The British Story, in M. Ferro, M. Brown, R. Cazals, O. Mueller (eds.), Meetings in No-Man's Land. Christmas and Fraternisation in the Great War, Constable \& Robinson Ltd., London 2007, pp. 13-76; T. Blom Crocker, The Christmas Truce. Myth, Memory, and the First World War, University Press of Kentucky, Lexington, KY 2015.

[23] P. Brown, Savage Enthusiasm. A History of Football Fans, Goal Post, Durham 2017, pp. 95103.

[24] Dunning, Murphy, Williams,1990, p. 32.

[25] M. Eve, Dentro l'Inghilterra. Ragioni e miti di un'identità, Marsilio, Venezia 1990. Pp. 106107.

[26] N. Elias, La civiltà delle buone maniere. La trasformazione dei costumi nel mondo aristocratico occidentale, Il Mulino, Bologna 2020 [1969], p. 211.

[27] E.J. Hobsbawm, Tradizioni e genesi dell'identità di massa in Europa, in Hobsbawm e Ranger (a cura di), 2002, p. 277.

[28] Le impressionanti immagini della folla che seguì la finale del 1923, vinta dai Wanderers sugli Hammers per 2-0, sono visualizzabili online sul canale Youtube della British Pathé. Cfr. World's Record Crowd, Wembley FA Cup (1923): 〈https://www.youtube.com/watch?v=c4NqYsFTr1Q> (ultimo accesso: 09.03.2021).

[29] Cfr. House of Commons Debates, 10 May 1923, vol. 163 cc2559-612559, 2559, consultabile online all'indirizzo $<$ https://api.parliament.uk/historic-hansard/commons/1923/may/10/wembleystadium-football-cup-tie $>$ (ultimo accesso: 15.03.2021)

[30] Cfr. P. Brown, Savage Enthusiasm. A History of Football Fans, Goal Post, Durham 2017, pp. 105-112.

[31] Dunning, Murphy, Williams, 1988, pp. 99-101.

[32] Cfr. a tale proposito R. Taylor, Football and its Fans. Supporters and their Relations with the Game, 1885-1985, Leicester University Press, London-New York 1992, p. 594. 
[33] P. Lanfranchi, I calciatori e il People's Game, in R. De Biasi (a cura di), You'll never walk alone. Mito e realtà del tifo inglese, Shake edizioni, Milano 2008, pp. 99-105.

[34] Cfr. su questi aspetti, in rapporto anche al contesto europeo, G. Silei, I fantasmi della golden age. Paura e incertezza nell'immaginario collettivo dell'Europa occidentale (1945-

1975), FrancoAngeli, Milano 2019, pp. 101-122.

[35] Cfr. P. Mignon, Supporters et Hooligans en Grande-Bretagne depuis 1871, in "Vingtième Siècle", Avril-Juin 1990, No. 26, pp. 42-43.

[36] Cfr. Silei, 2019, pp. 137-144.

[37] Cfr. Dunning, Murphy, Williams, 1988, pp. 148-150.

[38] P. Marsh, E. Rosser, R. Harré, The Rules of Disorder, Routledge \& Kegan Paul, London 1978.

[39] P. Mignon, Liverpool, ovvero “Addio alla Kop”, in De Biasi (a cura di), 2008, pp. 49-50.

[40] V. Marchi, Ultrà. Le sottoculture giovanili negli stadi d'Europa, Hellnation, Roma 2015, p. 37.

[41] Aggro Britain: 'Mindless violence' of the Bully Boys Worries Top Policemen, Daily Mirror, June 14, 1973. Sulla cultura aggro negli stadi inglesi e le sue regole si veda anche P. Marsh, Aggro. The Illusion of violence, Dent, London 1978.

[42] Dietschy, 2010, p. 398.

[43] Spurs and the Battle of Rotterdam, titolò ad esempio l'Evening Standard nel suo inserto sportivo.

[44] R. Ingham, Introduction and Foreground in R. Ingham, S. Hall, J. Clarke, P. Marsh, J. Donovan, Football Hooliganism. The Wider Context, Inter-Action Inprint, London 1978, p. 7.

[45] Affluent fans lead fighting, The Observer, 9 June 1985, p. 17. Sulla hoolilgan culture si soffermò anche il Times, cfr. David Nicholson-Lord, The alien society in our midst, The Times, May 31, 1985, p. 5.

[46] Per un profilo sociale e psicologico dei superhooligan cfr. G.W. Russell, Aggression in the Sports World. A Social Psychological Perspective, Oxford University Press, Oxford-New York 2008, pp. 150 e ss.

[47] Cfr. Eve, 1990, pp. 211-221.

[48] P. Murphy, J. Williams, E. Dunning, Football on trial. Spectator violence and development in the football world, Routledge, London 1990, p. 124.

[49] Si veda su questo R. Giulianotti, G. Armstrong, Comportamenti scorretti: gli hooligan, i media e la costruzione della notorietà, in De Biasi (a cura di), 2008, pp. 143-170. 
[50] B. Buford, I furiosi della domenica. Viaggio al centro della violenza ultrà, Longanesi, Milano 1992. Il titolo dell'edizione originale, uscita nel 1991, era Among the Thugs: The Experience, and the Seduction, of Crowd Violence.

[51] Si veda su questo, ad esempio, M. Layton, A. Pacey, Tracking the Hooligans. The History of Football Violence on the UK Rail Network, Stroud, Amberley 2016.

[52] Cfr. P. Murphy, J. Williams, E. Dunning, Football on Trial. Spectator violence and development in the football world, Routledge, London-New York 1990.

[53] Cfr. su tutti A. King, The End of the Terraces. The Transformation of English Football in the 1990s (Revised edition), Leicester University Press, London-New York 2002.

[54] D. Malcom, L. Walsh, A Survey of Arsenal and Aston Villa Fans, in Le public des stades d'Europe, Istututo Universitario Europeo 1993 cit. in Bromberger, 1999, p. 153.

[55] Sul "ribellismo" e mobilitazione delle tifoserie cfr. D. Brimson (ed.), Rebellion. The Inside Story of Football Protest Movement, lake, London 2006. Sul Football Activism in chiave comparata cfr. B. Garcia, J. Zheng (eds.), Foootball and Supporter Activism in Europe. Whose Game Is It?, Palgrave, Macmillan 2017.

[56] L'espressione è in P. Dietschy,.2010, p. 405. 